\title{
以芘一炔为基块的共价有机骨架材料的设计合成与表征
}

\author{
张婷婷 ${ }^{a}$ \\ 王海涛 ${ }^{a}$ \\ 马和平 $b$ 孙福兴 $b$ \\ 崔小强*, \\ 朱广山*,b \\ ( ${ }^{a}$ 吉林大学材料科学系 长春 130012) \\ ( ${ }^{b}$ 吉林大学无机合成与制备化学国家重点实验室＼cjkstart长春 130012)
}

\begin{abstract}
摘要 以四边形基块 1,3,6,8-四炔萠和二结点连接体 9,10-二溴葱通过 Sonogashira-Hagihara 偶联反应构建了一种多孔芳 香有机骨架材料 PAF-27. 差热重分析结果表明该材料具有一定的热稳定性, 在空气中可以稳定到 $320{ }^{\circ} \mathrm{C}$, 另外, 它在 一些常见的有机溶剂中能稳定存在，证明了该材料具有一定的化学稳定性. $\mathrm{N}_{2}$ 吸附结果表明，该材料的比表面积为 560 $\mathrm{m}^{2} / \mathrm{g}$, 平均孔径约为 $0.63 \mathrm{~nm}$. 由于该材料骨架结构中含有丰富的苯环和炔这样的基团, 加上材料本身较大的比表面积 和较窄的孔径分布, 将在气体的选择分离方面有潜在的应用前景.
\end{abstract}

关键词 多孔芳香有机骨架材料; Sonogashira-Hagihara 偶联反应; 热稳定性和化学稳定性; 较大的比表面积; 较窄的孔 径分布

\section{Construction and Characterization of Pyrene-alkyne Based Porous Frameworks}

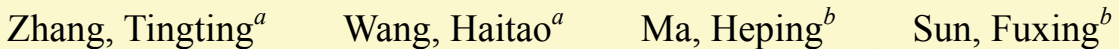 \\ Cui, Xiaoqiang*,a Zhu, Guangshan*,b \\ $\left({ }^{a}\right.$ Department of Materials Science, Jilin University, Changchun 130012) \\ ( ${ }^{b}$ State Key Laboratory of Inorganic Synthesis \& Preparative Chemistry, Jilin University, Changchun 130012)
}

\begin{abstract}
We report here a porous aromatic framework (PAF-27), which is constructed by a quadrilateral building block 1,3,6,8-tetraethynylpyrene with the linker 9,10-dibromoanthracene via Sonogashira-Hagihara coupling reaction. PAF-27 with plentiful phenyl rings in networks exhibits permanent porosity, high thermal and chemical stabilities, and excellent sorption abilities towards organic chemical pollutants at the saturated vapor pressure and room temperature. The structures of 1,3,6,8-tetrakis(4-(trimethylsilyl)ethynyl)pyrene, 1,3,6,8-tetraethynylpyrene and PAF-27 are firstly studied by ${ }^{1} \mathrm{H}$ NMR and ${ }^{13} \mathrm{C}$ CP/MAS NMR spectroscopy. As shown in the structure of PAF-27, all of the carbon signals with a chemical shift in the range of $\delta 120 \sim 140$ are observed, which are related to aromatic carbon atoms of building phenylene groups in the framework. Additionally, the structures with triple-bond linkages have been confirmed by the characteristic resonance of a triplebonded carbon with a corresponding peak at around $\delta 90$. IR spectra of initial monomer and final product can be found that the characteristic absorption peak for $\mathrm{C}-\mathrm{Br}$ at $577 \mathrm{~cm}^{-1}$ disappears, which gives an indication for the breakage of the $\mathrm{C}-\mathrm{Br}$ bonds of 9,10-dibromoanthracene. In addition, the intense absorption peak associated with alkynyl $\mathrm{C}-\mathrm{H}$ stretching vibration near $3280 \mathrm{~cm}^{-1}$ from the terminal alkyne groups vanishes. Meanwhile, a peak with low intensity is observed near $2169 \mathrm{~cm}^{-1}$ in the final product, which is assigned to the alkyne triple-bond stretching vibration mode. TGA analysis shows that PAF-27 is thermally stable up to $320{ }^{\circ} \mathrm{C}$ in air condition. In addition, the material also exhibits high chemical stability and cannot dissolve in any common organic solvents. $\mathrm{N}_{2}$ sorption results reveal that the BET surface area of PAF- 27 is $560 \mathrm{~m}^{2} / \mathrm{g}$ and the average pore size is about $0.63 \mathrm{~nm}$. Because of the rich aromatic rings, the large BET and narrow pore distribution, it will display relatively high sorption abilities for gases.

Keywords porous aromatic framework; Sonogashira-Hagihara coupling reaction; high thermal stability and chemical stability; the large BET and narrow pore distribution
\end{abstract}

\section{1 引言}

近年来，一些具有优良性质的多孔骨架材料 ${ }^{[1 \sim 5]}$ 被
许多课题组设计与合成. 由于该类材料具有较高的热稳 定性和化学稳定性、较大的比表面和可控的孔道尺寸被 广泛地用在气体的存储和选择性分离等方面 ${ }^{[6-8]}$. 该类 材料的设计与合成是通过选择以一定的基块和合适的

*E-mail: xqcui@jlu.edu.cn, zhugs@jlu.edu.cn

Received June 27, 2013; published September 16, 2013.

Project supported by the National Natural Science Foundation of China (Nos. 21075051, 21275064, 20831002), Program for New Century Excellent Talents in University (No. NCET-10-0433), National Basic Research Program of China (973 Program, No. 2012CB821700), Major International (Regional) Joint Research Project of NSFC (No. 21120102034).

项目受国家自然科学基金(Nos. 21075051, 21275064, 20831002)、新世纪优秀人才支持计划(No. NCET-10-0433)、国家重点基础研究发展计划(973 计 划, No. 2012CB821700)、重大国际(地区)合作研究(No. 21120102034)资助. 
连接体通过共价键按照常见的有机反应类型, 如：镍催 化的 Ullmann 偶联反应 ${ }^{[9-11]}$, 钯催化的 Suzuki 偶联反 应 $^{[12 \sim 14]}$, 钯催化的 Sonogashira-Hagihara ${ }^{[15 \sim 19]}$ 偶联反应 等构建起来的多孔骨架材料. 本课题组一直致力于多孔 骨架材料的设计与合成, 并报道了一些含丰富芳环的有 机骨架材料, 其中 PAF-1 以四节点的四溴苯甲烷为基块 通过 Ullmann 偶联反应构建成具有金刚石拓扑的多孔骨 架结构 ${ }^{[20]}$, PAF-11 以四结点的四溴苯甲烷为基块和 1,4对苯二硼酸通过 Suzuki 偶联反应构建成多孔有机骨架 材料, 以相对较高的比表面积对工业上有毒有害的芳香 化合物有较大的吸附效果 ${ }^{[21]}$.

本文通过 Sonogashira-Hagihara 偶联反应, 设计合 成了一种含萠和炔官能团的多孔有机骨架材料, 合成路 线如图 1 所示, 并通过 FTIR, ${ }^{1} \mathrm{H}$ NMR, ${ }^{13} \mathrm{C}$ NMR, TGA, XRD, SEM 及 $\mathrm{N}_{2}$ 吸附对其骨架结构与性质进行系统的 表征. 结果表明, PAF-27 具有良好的热稳定性、化学稳 定性、较大的比表面积和较窄的孔径分布, 使其在气体 分离等方面具有潜在的应用价值.

\section{2 结果与讨论}

\section{$2.1{ }^{1} \mathrm{H} \mathrm{NMR}$ 液体核磁谱图和 ${ }^{13} \mathrm{C} N \mathrm{NMR}$ 固体核磁谱图} 应用氢谱液体核磁来对聚合单体 $1,3,6,8$-四炔萠和
中间产物 1,3,6,8-四(4-(三甲基硅基)乙炔)萠的结构进行 分析. 如图 2(a)所示，在 1,3,6,8-四(4-(三甲基硅基)乙炔) 萠结构中, 萠核上的 3 种 $\mathrm{H}$ 分别出现在 $\delta 8.61,8.30$ 和 0.38 处, 且各组峰的积分面积比为 $2: 1: 18$; 如图 2(b) 所示, 在 1,3,6,8-四炔萠结构中, 萠核上的 3 种 $\mathrm{H}$ 分别出 现在 $\delta 8.68,8.39$ 和 3.66 处, 且各组峰的积分面积比为 $2: 1: 1$, 由此可见, 对于合成的 $1,3,6,8$-四炔萠的纯度 是比较高的. 应用碳谱固体核磁对 PAF-27 的结构进行 分析. 如图 2(c)所示, 在 PAF-27 结构中, 骨架中所有的 C 原子的化学位移均出现在 $\delta 120 \sim 140$, 而骨架中参键 的 $\mathrm{C}$ 原子的化学位移出现在 $\delta 90$ 处.

\section{2 红外光谱谱图}

应用红外光谱对 PAF-27 的结构进行分析. 如图 3 所示, 结合 $1,3,6,8$-四炔萠中端基炔中的参键 $\mathrm{C}-\mathrm{H}$ $\left(3280 \mathrm{~cm}^{-1}\right)$ 伸缩振动、参键的伸缩振动 $\left(2099 \mathrm{~cm}^{-1}\right)$ 和 9,10-二溴葸中 $\mathrm{C}-\mathrm{Br}\left(577 \mathrm{~cm}^{-1}\right)$ 的特征峰位置, 在 PAF-27 中可以看到, 端基炔中的参键 C-H $\left(3280 \mathrm{~cm}^{-1}\right)$ 伸缩振动特征峰的消失，同时，炔基中参键的伸缩振动 特征峰出现在 $2169 \mathrm{~cm}^{-1}$, 但强度极弱, 这样粗略地证 明了该聚合反应按预想的发生并进行的较完全. (a)<smiles>c1cc2ccc3cccc4ccc(c1)c2c34</smiles><smiles>O=[N+]([O-])c1ccccc1Br</smiles>
$120^{\circ} \mathrm{C}, 8 \mathrm{~h}$<smiles>C[Si](C)(C)C#Cc1cc(C#C[Si](C)(C)C)c2ccc3c(C#C[Si](C)(C)C)cc(C#C[Si](C)(C)C)c4ccc1c2c43</smiles>

(b)<smiles>C#Cc1cc(C#C)c2ccc3c(C#C)cc(C#C)c4ccc1c2c43</smiles><smiles>CCN(CC)CC(C)(C)c1ccccc1</smiles>
$60{ }^{\circ} \mathrm{C}, 30 \mathrm{~h}$<smiles>C#Cc1cc(C#C)c2ccc3c(C#C)cc(C#C)c4ccc1c2c43</smiles><smiles></smiles>

图 1 1,3,6,8-四芘炔(a)和 PAF-27 (b)的合成路线

Figure 1 Schematic illustration of synthesis of 1,3,6,8-tetraethynylpyrene (a) and PAF-27 (b) 

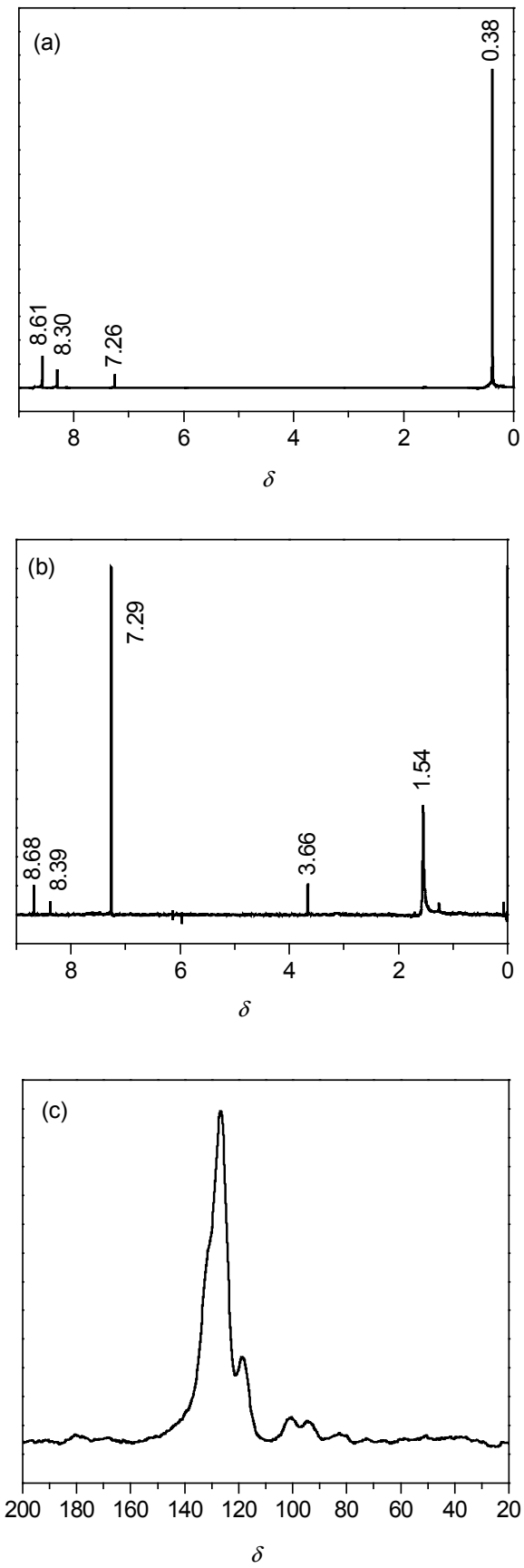

图 2 化合物 1,3,6,8-四(4-(三甲基硅基)乙炔)萠的液体氢谱核磁(a), 1,3,6,8-四炔萠的液体氢谱核磁(b)和 PAF-27 的固体碳谱核磁 (c) Figure $2 \quad{ }^{1} \mathrm{H}$ NMR spectrums of 1,3,6,8-tetrakis(4-(trimethylsilyl)ethynyl)pyrene (a), 1,3,6,8-tetra(ethynyl)pyrene (b) and ${ }^{13} \mathrm{C}$ CP/MAS NMR spectrum of PAF-27 (c)

\section{3 扫描电镜和粉末 $X$ 射线衍射}

应用扫描电镜对 PAF-27 的形貌进行表征. 如图 4 所示, PAF-27 呈现出絮状交织孔道, 同时结合它的粉末 XRD 谱图, 该谱图呈现出较宽的谱峰, 证明 PAF-27 为 无定形材料.

\section{4 稳定性能分析}

如图 5 所示, PAF-27 在室温下加热到 $320{ }^{\circ} \mathrm{C}$ 时开始
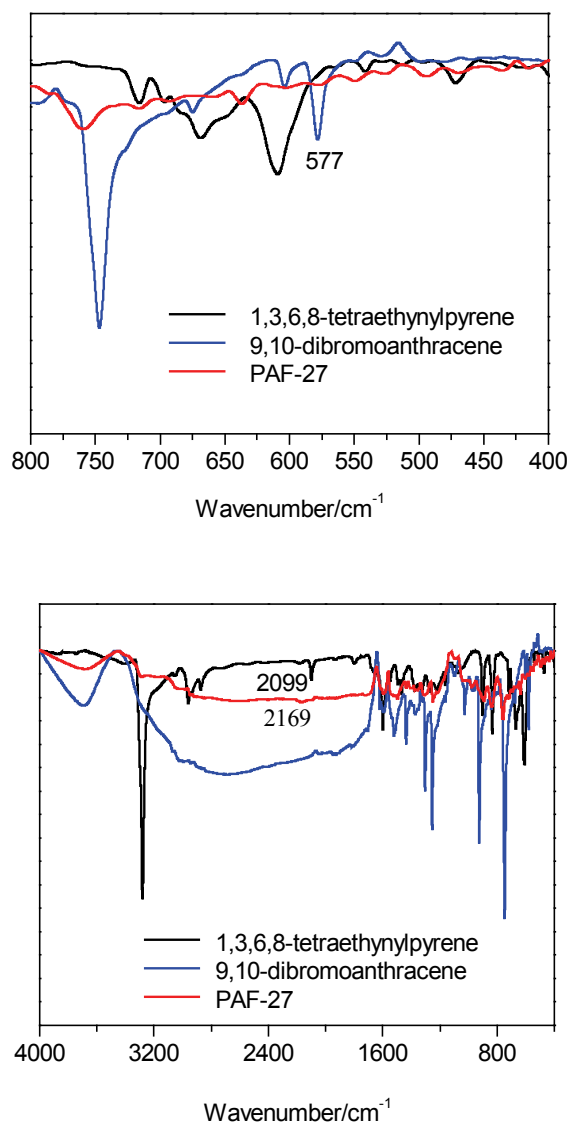

图 3 PAF-27 的红外光谱图谱, PAF-27 用红色曲线表示, 9,10-二澳葱 用蓝色曲线表示, $1,3,6,8$-四芘炔用黑色曲线表示

Figure 3 FTIR spectra for polymer networks of PAF-27 (red), 9,10-dibromoanthracene (blue) and 1,3,6,8-tetraethynylpyrene (black)

失重，证明该材料具有一定的热稳定性，除此之外， PAF-27 还具有良好的化学稳定性，能够在甲醇、乙醇、 丙酮、四氢呋喃、氯仿和 $N, N^{\prime}$-二甲基甲酰胺等常规有 机溶剂中稳定存在.

\section{5 孔道性质分析}

关于 PAF-27 孔道性质, 将活化处理后的样品在 77 $\mathrm{K}$ 时进行氮气吸附的测定. 如图 6 所示, PAF-27 表现出 I 型的吸附曲线特性, 应用 BET (Brunauer-Emmett-Teller)模型 得到 PAF-27 的表面积为 $560 \mathrm{~m}^{2} / \mathrm{g}$, 应用密度泛函理论 (DFT)测定的孔径分布，可知 PAF-27 的平均孔径约为 $0.63 \mathrm{~nm}$, 孔分布区域比较窄, 说明其具有规则的孔道结 构.

\section{3 结论}

选择四结点基块和二结点连接体通过 SonogashiraHagihara 偶联反应，合成了含有丰富苯环和炔基官能 团的多孔有机骨架材料 PAF-27, 该材料具有良好的热 稳定性和化学稳定性、较高的比表面积和较窄的孔径分 布，将在气体的吸附和选择性分离上有着潜在的应用前 
景
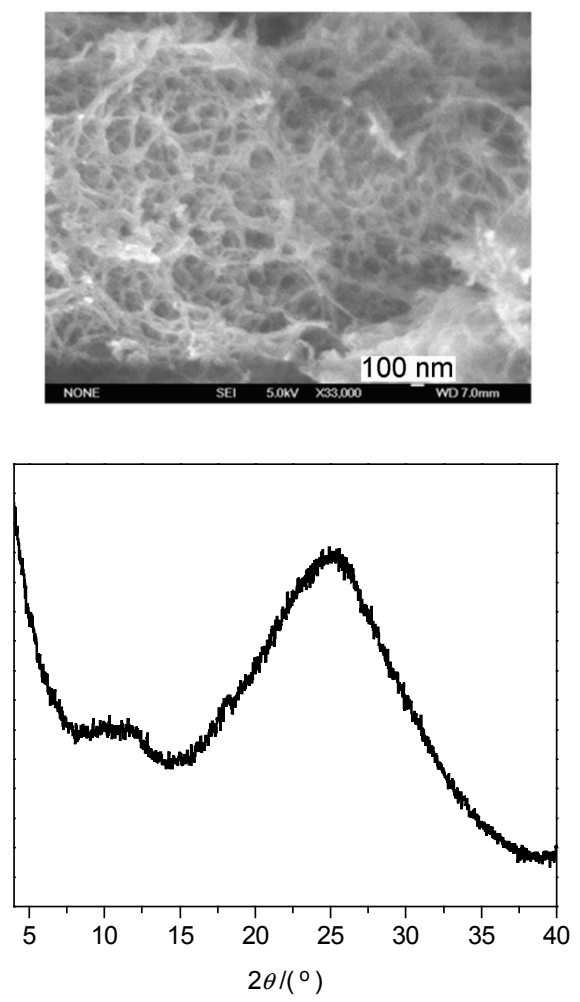

图 4 PAF-27 的扫描电镜和粉末 $X$ 射线衍射

Figure 4 SEM image and XRD of PAF-27

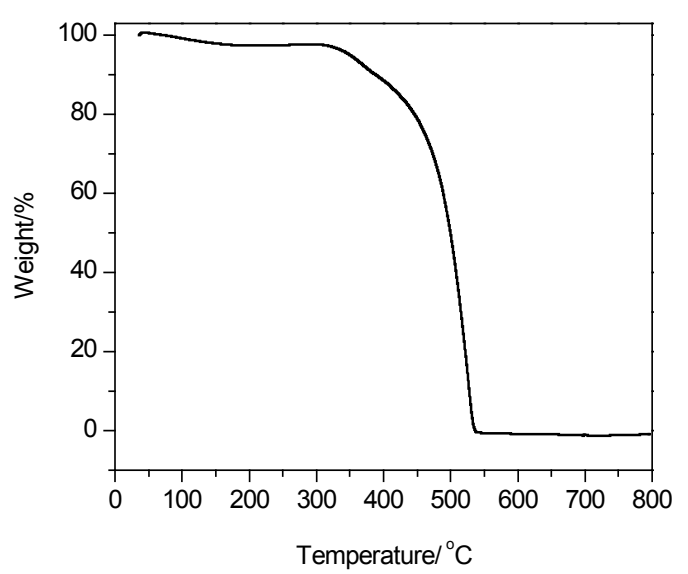

图 5 PAF-27 的热重曲线

Figure 5 TGA of PAF-27

\section{4 实验部分}

\subsection{1,3,6,8-四炔萠的合成路线}

1,3,6,8-四溴芘的制备: 将萠 $(1.0 \mathrm{~g}, 4.9 \mathrm{mmol}$ ) 溶于 硝基苯 $(20 \mathrm{~mL})$ 中, 待溶解后开始缓慢滴加液溴 $(1.12$ $\mathrm{mL}, 21.8 \mathrm{mmol}$ ), 最好在 $1 \mathrm{~h}$ 内滴加完成. 将反应体系升 温至 $120{ }^{\circ} \mathrm{C}$, 反应时间约 $6 \mathrm{~h}$, 溶液颜色开始变成土黄 色, 将得到的粗产品用乙醇超声清洗 3 次, 得到淡绿色 物质再放入真空干燥箱内干燥.

$1,3,6,8$-四炔萠的制备: 将 $1,3,6,8$-四溴萠 $(0.773 \mathrm{~g}$,
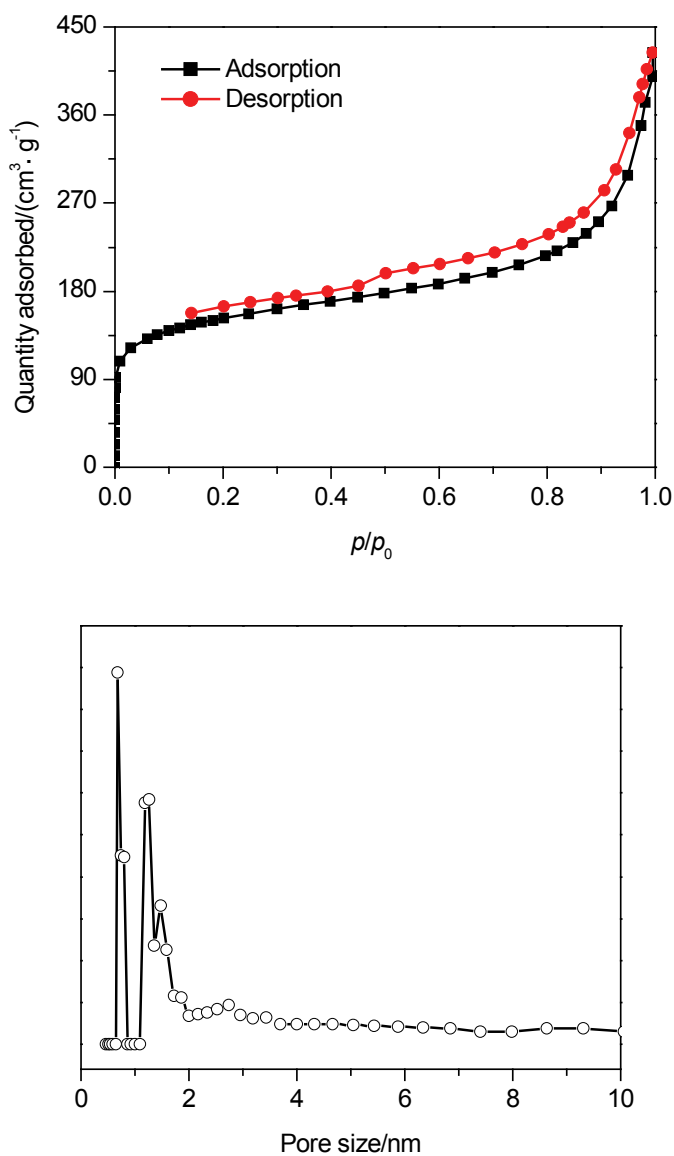

图 6 PAF-27 的 $\mathrm{N}_{2}$ 吸附-脱附曲线和孔径分布

Figure 6 Nitrogen gas adsorption-desorption isotherms and pore distribution

$1.5 \mathrm{mmol}), \mathrm{PdCl}_{2}\left(\mathrm{PPh}_{3}\right)_{2}(0.2 \mathrm{~g}, 28.5 \mathrm{mmol}), \mathrm{CuI}(0.068 \mathrm{~g}$, $35.7 \mathrm{mmol}), \mathrm{PPh}_{3}(0.157 \mathrm{~g}, 59.8 \mathrm{mmol})$ 于 $100 \mathrm{~mL}$ 二颈瓶 中, 加入完毕后反复置换气体 3 次, 用注射器将三甲基 硅基乙炔(TMSA, $1.68 \mathrm{~mL}$ )和三乙胺 $\left(\mathrm{Et}_{3} \mathrm{~N}, 20 \mathrm{~mL}\right.$ )注入 二颈瓶中, 反应温度为 $80{ }^{\circ} \mathrm{C}$, 时间为 $30 \mathrm{~h}$. 待反应完成 后, 将溶剂减压蒸干, 用 $\mathrm{CHCl}_{3}$ 溶解, 硅藻土过滤, 滤 液用 $\mathrm{Na}_{2}$ EDTA 洗涤, 最后用 $\mathrm{Mg}_{2} \mathrm{SO}_{4}$ 干燥, 旋干. 用石 油醚/二氯甲烷作为展开剂进行柱层析提纯. 将上述产 物 $0.39 \mathrm{~g}(0.6 \mathrm{mmol})$, 四丁基氟化铵(TBAF, $4.8 \mathrm{mmol}$ )在 $\mathrm{THF}$ 溶液中室温搅拌 $12 \mathrm{~h}$, 将得到的粗产物水洗 3 次, 过滤，得到 1,3,6,8-四芘炔.

\subsection{PAF-27 的合成路线}

将 1,3,6,8-四芘炔 $(0.2 \mathrm{~g}, 66.6 \mathrm{mmol}), 9,10$-二溴 $(0.22$ $\mathrm{g}, 66.0 \mathrm{mmol}) \mathrm{Pd}\left(\mathrm{PPh}_{3}\right)_{4}(20 \mathrm{mg}, 173 \mathrm{mmol})$, $\mathrm{CuI}(12 \mathrm{mg}$, $6.3 \mathrm{mmol})$ 于 $100 \mathrm{~mL}$ 二颈瓶中，加入完毕后反复置换气 体 3 次, 用注射器将三乙胺 $\left(\mathrm{Et}_{3} \mathrm{~N}, 15 \mathrm{~mL}\right.$ )和 $N, N^{\prime}$-二甲基 甲酰胺(DMF, $20 \mathrm{~mL}$ )注入反应瓶中, 反应温度为 $85{ }^{\circ} \mathrm{C}$, 时间为 $30 \mathrm{~h}$. 反应完毕后, 依次用二氯甲烷和水洗涤以 除去未反应的单体，过滤，依次用甲醇和四氢呋喃溶液 索氏提取 $48 \mathrm{~h}$, 真空抽干. 


\section{References}

[1] Pandey, P.; Farha, O. K.; Spokoyny, A. M.; Mirkin, C. A.; Kanatzidis, M. G.; Hupp, J. T.; Nguyen, S. T. J. Mater. Chem. 2011, 21, 1700.

[2] Liu, J.; Lukose, B.; Shekhah, O.; Arslan, H. K.; Weidler, P.; Gliemann, H.; Bräse, S.; Grosjean, S.; Godt, A.; Feng, X.; Müllen, K.; Magdau, I.-B.; Heine, T.; Wöll, C. Scientific Reports 2012, 2.

[3] Yaghi, O. M.; O'Keeffe, M.; Ockwig, N. W.; Chae, H. K.; Eddaoudi, M.; Kim, J. Nature 2003, 423, 705.

[4] Zhou, M.; Sun, L. B.; Liu, X. Q. J. Chem. Eng. Chin. Univ. 2012, 26, 858. (周满, 孙林兵, 刘晓勤, 高校化学工程学报, 2012, 26, 858.)

[5] Tian, G.; Zhu, G. S.; Fang, Q. R.; Xin, M. H.; Guo, X. D.; Xue, M.; Qiu, S. L. Chem. J. Chin. Univ. 2006, 27, 1020. (田歌, 朱广山, 方 千荣, 辛明红, 郭晓丹, 薛铭, 装式纶, 高等学校化学学报, 2006, 27, 1020.)

[6] Dawson, R.; Laybourn, A.; Clowes, R.; Khimyak, Y. Z.; Adams, D. J.; Cooper, A. I. Macromolecules 2009, 42, 8809.

[7] Yuan, Y.; Yan, Z.; Ren, H.; Liu, Q.; Zhu, G.; Sun, F. Acta Chim. Sinica 2012, 70, 1446. (元野, 间卓君, 任浩, 刘青英, 朱广山, 孙 福兴, 化学学报, 2012, 70, 1446.)

[8] Zhen, H. Y.; Luo, C.; Zhu, D. X.; Ye, H.; Liu, X. Acta Chim. Sinica 2008, 66, 557. (甄红宇, 罗渌, 朱德喜, 叶辉, 刘旭, 化学学报, 2008, 66, 557.)

[9] Liang, Y.; Li, J. H. Chin. J. Org. Chem. 2005, 25, 147. (梁云，李金 恒, 有机化学, 2005, 25, 147.)

[10] Ren, H.; Ben, T.; Sun, F.; Guo, M.; Jing, X.; Ma, H.; Cai, K.; Qiu,
S.; Zhu, G. J. Mater. Chem. 2011, 21, 10348.

[11] Lu, W.; Yuan, D.; Zhao, D.; Schilling, C. I.; Plietzsch, O.; Muller, T.; Brase, S.; Guenther, J.; Blumel, J.; Krishna, R.; Li, Z.; Zhou, H.-C. Chem. Mater. 2010, 22, 5964.

[12] Jia, J.; Sun, F.; Fang, Q.; Liang, X.; Cai, K.; Bian, Z.; Zhao, H.; Gao, L.; Zhu, G. Chem. Commun. 2011, 47, 9167.

[13] Lü, X.; Qian, Y. Chin. J. Org. Chem. 2011, 31, 82. (闾新明，钱鹰， 有机化学, 2011, 31, 82.)

[14] Chen, L.; Honsho, Y.; Seki, S.; Jiang, D. L. J. Am. Chem. Soc. 2010, 132,6742 .

[15] Stöckel, E.; Wu, X.; Trewin, A.; Wood, C. D.; Clowes, R.; Campbell, N. L.; Jones, J. T. A.; Khimyak, Y. Z.; Adams, D. J.; Cooper, A. I. Chem. Commun. 2009, 212.

[16] Jiang, J.-X.; Laybourn, A.; Clowes, R.; Khimyak, Y. Z.; Bacsa, J.; Higgins, S. J.; Adams, D. J.; Cooper, A. I. Macromolecules 2010, 43 , 7577.

[17] Farha, O. K.; Spokoyny, A. M.; Hauser, B. G.; Bae, Y. S.; Brown, S. E.; Snurr, R. Q.; Mirkin, C. A.; Hupp, J. T. Chem. Mater. 2009, 21, 3033.

[18] Sasaki, T.; Tour, J. M. Org. Lett. 2008, 10, 897.

[19] Lipshutz, B. H.; Chung, D. W.; Rich, B. Org. Lett. 2008, 10, 3793.

[20] Ben, T.; Ren, H.; Ma, S.; Cao, D.; Lan, J.; Jing, X.; Wang, W.; Xu, J.; Deng, F.; Simmons, J. M.; Qiu, S.; Zhu, G. Angew. Chem., Int. Ed. 2009, 48, 9457.

[21] Yuan, Y.; Sun, F.; Ren, H.; Jing, X.; Wang, W.; Ma, H.; Zhao, H.; Zhu, G. J. Mater. Chem. 2011, 21, 13498.

(Qin, X.) 